Revue d'histoire de l'Amérique française

REYUE D.HISTOIRE DE L'AMÉRIQUE FRANÇAISE

\title{
BIRON, Michel, François DUMONT et Élisabeth \\ NARDOUT-LAFARGE avec la collaboration de \\ Martine-Emmanuelle LAPOINTE, Histoire de la littérature \\ québécoise (Montréal, Boréal, 2007), 689 p.
}

\section{Damien-Claude Bélanger}

Volume 61, numéro 3-4, hiver-printemps 2008

Dossier Québec

URI : https://id.erudit.org/iderudit/019130ar

DOI : https://doi.org/10.7202/019130ar

Aller au sommaire du numéro

Éditeur(s)

Institut d'histoire de l'Amérique française

ISSN

0035-2357 (imprimé)

1492-1383 (numérique)

Découvrir la revue

Citer ce compte rendu

Bélanger, D.-C. (2008). Compte rendu de [BIRON, Michel, François DUMONT et Élisabeth NARDOUT-LAFARGE avec la collaboration de Martine-Emmanuelle LAPOINTE, Histoire de la littérature québécoise (Montréal, Boréal, 2007), 689 p.] Revue d'histoire de l'Amérique française, 61(3-4), 565-568.

https://doi.org/10.7202/019130ar d'utilisation que vous pouvez consulter en ligne. 
BIRON, Michel, François DUMONT et Élisabeth NARDOUT-LAFARGE avec la collaboration de Martine-Emmanuelle LAPOINTE, Histoire de la littérature québécoise (Montréal, Boréal, 2007), 689 p.

On n'avait pas publié une grande synthèse de la littérature québécoise depuis l'Histoire de la littérature française du Québec de Pierre de Grandpré (1967), dont les quatre magnifiques volumes reflétaient clairement l'énergie et le dynamisme des années 1960. Malgré les qualités de cet ouvrage, il était temps, quelque quarante ans plus tard, de produire une nouvelle synthèse de la littérature québécoise. Une équipe de quatre spécialistes a relevé ce défi et a produit un excellent volume qui trace un portrait de la littérature québécoise de ses origines à nos jours.

L'ouvrage de Michel Biron, François Dumont, Élisabeth Nardout-Lafarge et Martine-Emmanuelle Lapointe est divisé en cinq grandes sections chronologiques. Chaque section se compose de plusieurs courts chapitres consacrés soit à des genres littéraires, soit à des écrivains, soit à des œuvres, soit à des écoles. Les auteurs utilisent une définition de la vie littéraire qui comprend non seulement le roman et la poésie, mais aussi le récit de voyage, la correspondance et l'essai. Cette conception élargie de la littérature est surtout évidente dans les sections portant sur les écrits d'avant 1900, période où il s'est publié assez peu d'œuvres de fiction au Québec.

La première section porte sur les écrits de la Nouvelle-France. Les auteurs insistent d'ailleurs sur le caractère fondateur des écrits de Jacques Cartier et notent que la littérature du Régime français visait un public français et européen. Cette tendance se renverse après la Conquête, alors que la littérature canadienne-française se tourne presque totalement vers un public d'ici. Plusieurs des grandes caractéristiques de la littérature canadienne-française se précisent d'ailleurs dans la section suivante de la 
synthèse, qui porte sur les années 1763-1895. À partir du XIX ${ }^{\mathrm{e}}$ siècle, la littérature canadienne-française se définit dans le cadre d'un projet national. La question nationale sera d'ailleurs omniprésente dans les débats littéraires.

Biron, Dumont, Nardout-Lafarge et Lapointe ont divisé le $\mathrm{xx}^{\mathrm{e}}$ siècle, auquel ils consacrent la majeure partie de leur synthèse, en trois sections: 1895 à 1945, 1945 à 1980 et 1980 à nos jours. Pour ces chercheurs, la création de l'École littéraire de Montréal marque le début du xx $x^{\mathrm{e}}$ siècle littéraire au Québec. Les premières décennies de ce siècle sont marquées par la querelle esthétique et idéologique qui oppose les tenants du traditionalisme littéraire à ceux qui préconisent une littérature plus moderne. Plus tard, durant l'entre-deux-guerres, une génération de femmes s'impose sur la scène littéraire, particulièrement dans le milieu de la poésie. C'est à cette époque qu'une véritable tradition d'écriture se met en place au Québec. La Deuxième Guerre mondiale bouleverse la vie littéraire du Québec. Elle ouvre la porte à de nouvelles esthétiques et, selon les auteurs, «fait entrer symboliquement le monde extérieur dans la culture canadienne-française» (p. 274). On voit alors apparaître les premiers signes d'une véritable révolution culturelle.

En effet, bien que les années 1960 marquent une rupture symbolique dans l'évolution de la littérature québécoise, Biron, Dumont, NardoutLafarge et Lapointe soulignent qu'il ne faut pas réduire la Révolution tranquille uniquement à cette décennie. Une infrastructure littéraire se met en place durant les années 1950 et la littérature québécoise acquiert une nouvelle autonomie. Durant la décennie suivante, la littérature québécoise se caractérise par un fort degré d'unité, qui ne tient pas tant à des considérations esthétiques qu'aux discours qui accompagnent les œuvres. Les écrivains des années 1960 se retrouvent à l'avant-scène du mouvement nationaliste et jouissent d'une visibilité sans précédent dans les débats politiques. Leur unité et leur influence s'estompent toutefois au cours des années 1970. Plusieurs d'entre eux chercheront alors à transgresser les conceptions classiques de la littérature au nom de la contre-culture, du formalisme ou du féminisme.

La littérature québécoise entre dans l'ère du pluralisme à partir de 1980. Les désillusionnements qui accompagnent l'échec référendaire vont d'ailleurs contribuer à l'éclatement du champ littéraire. La référence nationale s'estompe alors au profit de questionnements plus diversifiés, notamment ceux liés à la sexualité et à la migration. L'émergence de l'écriture migrante constitue en effet une des transformations les plus 
importantes de la littérature québécoise à la fin du $\mathrm{xx}^{\mathrm{e}}$ siècle. L'institutionnalisation de la littérature québécoise s’achève à cette époque. Les écrivains peuvent alors bénéficier d'un nombre croissant de subventions et les œuvres québécoises sont enseignées à tous les niveaux du système d'éducation. Cependant, les dernières décennies du $\mathrm{xx}^{\mathrm{e}}$ siècle ne marquent pas pour autant l'apothéose de cette littérature. Au cours de ces années, le domaine littéraire se subdivise progressivement en secteurs relativement indépendants les uns des autres. Selon les auteurs, «le phénomène de spécialisation peut être perçu comme une conséquence du décentrement de la littérature et de la perte d'influence de l'écrivain. Celui-ci n'aurait de véritable influence que dans un milieu plus ou moins restreint qui lui assurerait sa légitimité au sein de l'institution littéraire» (p. 591). La littérature québécoise serait la victime d’une certaine marginalisation, observable actuellement à l'échelle de l'Occident. Biron, Dumont, Nardout-Lafarge et Lapointe reprennent la formule de Pierre Nora selon laquelle nous vivons actuellement le «deuil éclatant de la littérature». Au Québec, disent-ils, «ce deuil est d'autant plus ressenti qu'une telle situation survient immédiatement après une période où la littérature a été, plus qu'ailleurs, un vecteur de l'identité nationale» (p. 629). L'ouvrage se termine ainsi avec une note de pessimisme qui tranche nettement avec l'optimisme quasi triomphaliste de l'Histoire de la littérature française $d u$ Québec de Pierre de Grandpré. Autres temps, autres mœurs.

L'inclusion d'écrivains anglo-québécois constitue une des grandes nouveautés de cette histoire littéraire. Les anglophones ont traditionnellement été exclus des travaux portant sur la littérature québécoise, où les adjectifs "québécois» et "canadien-français» ont longtemps été synonymes. L'ouvrage renverse cette tendance et consacre trois chapitres aux écrivains anglophones de Montréal. Malheureusement, ces trois chapitres portent essentiellement sur les écrivains actifs durant la deuxième moitié $\mathrm{du} \mathrm{xx}^{\mathrm{e}}$ siècle et ne contiennent qu'un bref paragraphe sur l'œuvre de Stephen Leacock, dont les célèbres récits humoristiques étaient lus dans tout le monde anglophone. Andrew Macphail, qu’on associe généralement à la littérature des provinces maritimes, mais qui a habité le Québec pendant plus de quarante ans, est entièrement exclu de cette Histoire de la littérature québécoise. Pourtant, Macphail a joué un rôle de premier plan dans le développement de la littérature canadienne-anglaise. C'est grâce à son œuvre critique que les poètes Marjorie Pickthall et John McCrae ont fini par être connus du grand public et c'est Macphail qui effectua la première traduction anglaise de Maria Chapdelaine. 
Ces oublis n'enlèvent rien à la qualité de l'Histoire de la littérature québécoise. L'ouvrage aborde la littérature québécoise sous un angle pluraliste et l'insère dans un contexte international. Le rapport ambigu et parfois turbulent entre les écrivains québécois et la France est d'ailleurs décrit avec une grande finesse. Cet ouvrage présente un intérêt particulier pour les spécialistes de l'histoire intellectuelle et culturelle du Québec, qui ont parfois tendance à négliger le champ littéraire. En effet, comme le démontrent si clairement les auteurs de cette synthèse, l'histoire littéraire est intimement liée à l'évolution intellectuelle et culturelle du Québec. Les approches historique et littéraire se complètent et se renforcent et il serait sans doute avantageux pour les deux disciplines qu'une plus grande collaboration s'installe entre elles.

DAMIEN-CLAUDE BÉLANGER Département d'histoire Université d'Ottawa

BIZIMANA,Aimé-Jules, De Marcel Ouimet à René Lévesque. Les correspondants de guerre canadiens-français durant la Deuxième Guerre mondiale (Montréal, vlb éditeur, coll. «Études québécoises », 2007), $369 \mathrm{p}$.

Comment les Canadiens francophones ont-ils suivi ce qui arrivait outremer à leurs soldats? Le titre même de l'ouvrage de référence sur le sujet du reportage de guerre, The First Casualty: The War Correspondent as Hero and Myth-Maker from Crimea to Kosovo de Philip Knightley, trahit d'emblée son parti pris et Bizimana ne se prononce pas sur l'objectivité des éminents journalistes québécois qui rendaient compte, sur tous les fronts, de la guerre menée par les Alliés contre Mussolini, Hitler et l'empereur Hirohito. D’ailleurs, pratiquement intégrés de fait aux forces armées, les reporters de guerre en uniforme et à la merci de l'armée canadienne pour leurs déplacements et leur subsistance en campagne étaient les agents minutieusement contrôlés d'un effort de guerre qui dépendait largement de l'adhésion civique à l'effort de guerre national, et ce, d'autant plus que l'enthousiasme des Canadiens francophones pour cette cause était quelque peu incertain.

Bizimana a soigneusement séparé son traitement à moitié biographique des correspondants de guerre canadiens-français d'un chapitre sur la gestion de la censure par Ottawa et, de manière nettement plus significative, par ses alliés de Londres et Washington. Personne ne sera surpris 